\title{
Guidelines for molecular karyotyping in constitutional genetic diagnosis
}

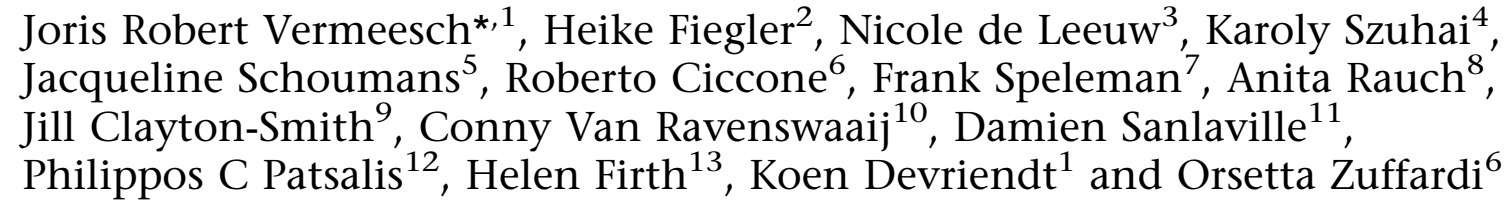

${ }^{1}$ Center for Human Genetics, University Hospital Gasthuisberg, Leuven, Belgium; ${ }^{2}$ The Wellcome Trust Sanger Institute, Hinxton, Cambridgeshire, UK; ${ }^{3}$ Department of Human Genetics, Nijmegen Centre for Molecular Life Sciences, Radboud University Nijmegen Medical Centre, Nijmegen, The Netherlands; ${ }^{4}$ Department of Molecular Cell Biology, Leiden University Medical Centre, Leiden, The Netherlands; ${ }^{5}$ Department of Molecular Medicine and Surgery, Karolinska Institute, Karolinska University Hospital Solna, Stockholm, Sweden; ${ }^{6}$ Biologia Generale e Genetica Medica, Università di Pavia, Pavia, Italy; ${ }^{7}$ Center for Medical Genetics, Ghent University, Ghent, Belgium; ${ }^{8}$ Institute of Human Genetics, Friedrich-Alexander University Erlangen-Nuremberg, Erlangen, Germany; ${ }^{9}$ Academic Unit of Medical Genetics, St Mary's Hospital, Manchester, UK; ${ }^{10}$ Department of Genetics, University Medical Center, Groningen, The Netherlands; ${ }^{11}$ Cytogenetic Department, Hospices Civils de Lyon, Edouard Herriot Hospital, and Claude Bernard University, Lyon, France; ${ }^{12}$ Department of Cytogenetics, The Cyprus Institute of Neurology and Genetics, Nicosia, Cyprus and ${ }^{13}$ Department of Clinical Genetics, Addenbrooke's Hospital, Cambridge, UK

Array-based whole genome investigation or molecular karyotyping enables the genome-wide detection of submicroscopic imbalances. Proof-of-principle experiments have demonstrated that molecular karyotyping outperforms conventional karyotyping with regard to detection of chromosomal imbalances. This article identifies areas for which the technology seems matured and areas that require more investigations. Molecular karyotyping should be part of the genetic diagnostic work-up of patients with developmental disorders. For the implementation of the technique for other constitutional indications and in prenatal diagnosis, more research is appropriate. Also, the article aims to provide best practice guidelines for the application of array comparative genomic hybridisation to ensure both technical and clinical quality criteria that will optimise and standardise results and reports in diagnostic laboratories. In short, both the specificity and the sensitivity of the arrays should be evaluated in every laboratory offering the diagnostic test. Internal and external quality control programmes are urgently needed to evaluate and standardise the test results between laboratories.

European Journal of Human Genetics (2007) 15, 1105-1114; doi:10.1038/sj.ejhg.5201896; published online 18 July 2007

Keywords: best practice guidelines; molecular karyotype; array $\mathrm{CGH}$; copy number changes; genome-wide screening

\footnotetext{
${ }^{*}$ Correspondence: Professor JR Vermeesch, Centre for Human Genetics, University Hospital Gasthuisberg, Herestraat 49, 3000 Leuven, Belgium. Tel: + 3216 345941; Fax: + 3216 346060;

E-mail: Joris.Vermeesch@med.kuleuven.be

Received 2 March 2007; revised 9 June 2007; accepted 15 June 2007; published online 18 July 2007
}

\section{Introduction}

Following the discovery that humans carry 46 chromosomes and the association of different aneuploidies with specific phenotypes in the late 1950s, karyotyping was rapidly introduced as a tool for the aetiological diagnosis of patients with mental retardation (MR) and birth defects. ${ }^{1-5}$ Initially, chromosome studies were performed using simple 
staining techniques which only allowed the detection of entire groups of chromosomes. The degree of precision was then increased in the 1970s with the introduction of chromosome banding techniques. These techniques enabled the detection of individual chromosomes ${ }^{6,7}$ and led to the identification of multiple syndromes associated with specific chromosomal imbalances. ${ }^{8,9}$ Although chromosomal karyotyping allows a genome-wide detection of large chromosomal abnormalities and translocations, it has a number of inherent limitations: (1) it takes between 4 and 10 days to culture the cells, visualise chromosomes and perform the analysis; (2) the resolution of a karyotype is limited to $5-10 \mathrm{Mb}$ depending on (i) the location in the genome, (ii) the quality of the chromosome preparation and (iii) the skill and experience of the cytogeneticist; and (3) it requires skilled technicians to perform a Giemsa-banded karyotype analysis, which increases employment costs and can lead to organisational difficulties in small laboratories.

With the introduction of fluorescence in situ hybridisation (FISH) the detection of submicroscopic chromosomal imbalances became possible. In FISH, labelled DNA probes are hybridised to nuclei or metaphase chromosomes to detect the presence, number and location of small (submicroscopic) regions of chromosomes. FISH is routinely used to identify, confirm and characterise chromosomal abnormalities or confirm the clinical suspicion of known microdeletion syndromes. ${ }^{10}$ Also, more recently, other molecular methods, such as quantitative PCR (Q-PCR) and multiplex ligation-dependent probe amplification (MLPA), have been developed to detect accurately submicroscopic copy number variations (CNVs) with the possibility to interrogate multiple targets within one experiment (reviewed by Sellner and Taylor ${ }^{11}$ ). However, all these methods are targeted and can only screen individual DNA targets rather than the entire genome.

To overcome this problem, multicolour FISH-based karyotyping (SKY, MFISH and COBRA FISH) was developed, which enables simultaneous detection of all chromosomes. ${ }^{10,12,13}$ Another technology allowing the genomewide detection of copy number aberrations was introduced in 1992 and termed comparative genomic hybridisation (CGH) ${ }^{14}$ In $\mathrm{CGH}$, test and reference genomic DNAs are differentially labelled with fluorochromes and then cohybridised onto normal metaphase chromosomes. Following hybridisation, the chromosomes are scanned to measure the fluorescence intensities along the length of the normal chromosomes to detect intensity ratio differences that subsequently pinpoint to genomic imbalances. Overall, the resolution at which copy number changes can be detected using these techniques are only slightly higher as compared to conventional karyotyping ( $>3 \mathrm{Mb}$ ) and all experiments are labour intensive and time consuming.

By replacing metaphase chromosomes with mapped DNA sequences or oligonucleotides arrayed onto glass slides as the hybridisation targets, these limitations could be overcome. Following hybridisation of differentially labelled test and reference genomic DNAs to the target sequences on the microarray, the slide is scanned to measure the fluorescence intensities at each target on the array. The normalised fluorescent ratio for the test and reference DNAs is then plotted against the position of the sequence along the chromosomes. Gains or losses across the genome are identified by values increased or decreased from a 1:1 ratio ( $\log 2$ value of 0 ), and the detection resolution now only depends on the size and the number of targets on an array and the position of these targets (their distribution) on the genome. This methodology was first described in 1997 and is termed matrix or array CGH. ${ }^{15,16}$ Array CGH has initially been employed to analyse copy number changes in tumours with the aim to identify genes involved in the pathogenesis of cancers. ${ }^{17,18}$ More recently, however, this methodology has been optimised and applied to detect unbalanced constitutional rearrangements. ${ }^{19,20}$

Genome-wide screening for CNVs in a clinical genetic context at a defined high resolution has been termed molecular karyotyping and includes technologies such as array CGH or hybridisation methods in which control and sample are hybridised separately. ${ }^{21,22}$ Several studies have demonstrated the potential value of genomic arrays in the diagnosis of thus far unexplained developmental/MR disorders. It has been proposed that in analogy with the introduction of chromosome karyotyping in the early 1960s, genomic arrays should now be implemented as a genetic diagnostic service and several genetic centres already offer molecular karyotyping to patients. ${ }^{23,24}$ However, the introduction of molecular karyotyping in genetic services raises many clinical as well as technical questions: for which disorders is the test useful? What is the clinical setting in which the tests should be requested, performed and interpreted? What are the positive and negative predictive values? What are the genotype/phenotype relationships? Considering that molecular karyotyping can be performed in different ways, using different array platforms and software, the outcome of a genomic array experiment on patient DNA may be very different in different laboratories or even within a single laboratory. Hence, there is a need to define quality standards as well as guidelines for patient reports. This article aims to address the clinical utility and validity of molecular karyotyping and provides a framework on how to introduce the array technology in clinical genetics (ACCE; http://www.cdc.gov/ genomics/gtesting/ACCE.htm, Burke et al, 2002 25 ).

\section{Clinical utility \\ Postnatal diagnosis of developmental delay/MR and/or congenital anomalies \\ MR with or without congenital malformations occurs in $\sim 3 \%$ of the population and is aetiologically hetero-}


geneous. ${ }^{26}$ Chromosomal anomalies as detected by conventional karyotyping are one of the most common recognised causes, accounting for an estimated $10 \%$ of $\mathrm{MR} \cdot{ }^{27}$ In about $50 \%$ of patients with MR, the aetiology remains unknown.

Nevertheless, an aetiological diagnosis is important for many reasons. A precise diagnosis may, for example, lead to prevention or even an earlier detection of some pathogenic signs (eg obesity in Prader-Willi patients, Wilms tumour in Wilms tumour, aniridia, genitourinary anomalies mental retardation (WAGR) syndrome) or may enable improved medical care for the individual (eg echocardiogram if a gene implicated in congenital heart disease is involved). Moreover, a diagnosis often is essential for genetic counselling and reproductive choices of the individual and his/her family. For parents, insight in the cause of a handicap will lead to reduced uncertainty and may alleviate feelings of guilt. ${ }^{28}$ Since in $50 \%$ of patients no aetiological diagnosis can be made, ${ }^{29}$ families may seek advice from several specialised physicians and undergo multiple diagnostic tests. This leads to a 'diagnostic odyssey' of often repeated testing for many different conditions. ${ }^{23}$ An early diagnosis will avoid the anxiety and strain put on a patient and his family by the current procedures. These frequently involve multiple hospital appointments and repeated and often invasive investigations, some of which (eg magnetic resonance imaging under sedation) are not entirely risk-free.

Several studies have demonstrated the efficacy of arraybased methods to detect apparent pathogenic imbalances in both children and adults with MR and multiple congenital anomalies (MCA), but normal conventional cytogenetic analyses. These studies have been performed using platforms of increasing resolution. The first reports described the application of chromosome-specific arrays ${ }^{30}$ and large insert clone arrays covering the entire human genome at $1 \mathrm{Mb},{ }^{19,20,24,31,32}$ or more; recently, full-tiling path resolution. ${ }^{33}$ In addition, oligo-based array platforms are now being increasingly applied in these studies. ${ }^{23}$ While a comparison of the detection rate of pathogenic imbalances in these studies is confounded by different patient selection criteria, overall $7-11 \%$ of patients were diagnosed with cryptic pathogenic submicroscopic chromosomal imbalances. In addition, in patient cohorts that have not previously screened for subtelomeric imbalances, about $5 \%$ of subtelomeric imbalances are detected. This number is roughly in concordance with the numerous reports on subtelomeric screens in these types of patient cohorts. ${ }^{34}$ The detection rate of pathogenic imbalances appears to increase slightly by using higher resolution arrays. High-resolution screens of MCA/MR patients with MCA/MR using either full-tiling large insert clone or oligo arrays found that $20 \%$ of all detected imbalances were smaller than $1 \mathrm{Mb}$, indicating that higher resolutions screens will improve the diagnostic yield, but also add complexity due to the need to differentiate pathogenic from normal CNV at this level of resolution. ${ }^{23,33}$ Thus, with regard to the diagnosis of unexplained MCA/MR, the criteria of clinical utility for molecular karyotyping in this specific setting certainly is fulfilled. General experience suggests to request this investigation with the same criteria used to request cytogenetic investigations.

In addition to the diagnosis of patients without visible chromosomal anomalies, molecular karyotyping can also reveal unsuspected imbalances in patients with visible chromosomal imbalances. In particular, apparently balanced translocations in patients with abnormal phenotypes may hide deletions both at the breakpoint and elsewhere in the genome. ${ }^{35,36}$ Preliminary data demonstrated that other rearrangements also, such as ring chromosomes, may be more complex than anticipated ${ }^{37}$ (Rossi et al, submitted). Hence, it is advised to investigate these rearrangements through whole-genome arrays.

\section{Prenatal diagnosis}

In analogy with conventional prenatal karyotyping, it is tempting to introduce molecular karyotyping in a prenatal setting. The key issues are the speed (no culturing is required and automation of certain steps in the procedure is possible) and the higher resolution with molecular compared to conventional karyotyping. A number of proof-of-principle clinical studies are currently ongoing. ${ }^{38-41}$ However, there are a number of potential pitfalls that have to be avoided.

Most prenatal testing is carried out in the context of advanced maternal age or increased risk for Down syndrome based on ultrasound tests or serum markers. In this context, many laboratories have already moved from conventional karyotyping towards MLPA, Q-PCR or interphase FISH, either directed at detecting only trisomy 21 or in combination with detection of aneuploidies for chromosomes 13, 18, $\mathrm{X}$ and $\mathrm{Y}$. However, in contrast to conventional karyotyping or FISH analysis for established loci, genome-wide array analysis interrogates the entire genome. Array CGH will obviously detect any of those whole-chromosome alterations with the possibility to detect mosaic alterations (see later sections). In addition to the detection of imbalances resulting in well-established developmental disorders, molecular karyotyping will detect imbalances in regions for which we do not know the phenotypic consequences yet. Although benign DNA copy number variants are currently being inventorised, assessment of their clinical relevance requires further study. Also, for each new imbalance that has not been reported previously, phenotypic consequences and penetrance may be difficult to predict in the absence of extensive family data and genotyope/phenotype correlations. It is difficult to envisage how this specific setting could benefit from the introduction of molecular karyotyping, particularly with regard to its increased resolution and thus the potential to detect imbalances that at present cannot be 
interpreted. A possible solution to this problem might be the development of arrays with a limited number of wellknown targets.

In the case of prenatal karyotyping following the detection of malformations on ultrasound examinations, the a priori chance to detect a causal aberration is much higher, and especially in the presence of specific phenotypic anomalies, the interpretation of an abnormal result may be facilitated by the molecular analyses. Potentially, more extensive pre- and post-testing counselling will be needed to ensure that imbalances which cannot be interpreted are not perceived as true anomalies by parents and physicians. However, clinical geneticists are already familiar with this problem when facing most of counselling cases with de novo supernumerary marker chromosomes or de novo apparently balanced translocations. Carefully designed clinical studies, initially in a research context, are warranted to establish practical guidelines.

A de novo apparently balanced chromosome rearrangement is associated with a $6.7 \%$ risk of a congenital anomaly in the child, some of which will be related to a submicroscopic imbalance. ${ }^{42}$ Currently it is not clear what percentage of these children do harbour submicroscopic imbalances, and molecular karyotyping may aid in the unmasking of these translocation-associated imbalances. $^{35,36,42}$ Further research in this area is strongly recommended. Likewise for extra structurally abnormal chromosomes, molecular karyotyping has the potential to determine the size and the presence of euchromatic regions more precisely. However, as for other chromosomal imbalances, currently genotype/phenotype data on small, supernumerary marker chromosomes are not sufficient to allow accurate interpretation in all instances. ${ }^{43}$ Hence, in this area also more research is needed.

\section{Infertility and recurrent abortion}

While molecular karyotyping can reveal imbalances in the genome, to date it cannot detect balanced rearrangements without the separation of the derivative chromosomes away from the rest of the genome before the analysis ${ }^{44-46}$ - a procedure that is not easily amenable in a diagnostic setting. Since chromosomal translocations are a significant cause of infertility, conventional karyotyping remains the key diagnostic test for this indication. Recurrent miscarriages are often caused by the unbalanced transmission of a chromosomal translocation in one of the parents. Subtelomeric analysis using FISH may also be used to investigate for the presence of cryptic translocations although only 1 in 50 couples with repeated abortions had been reported harbouring such a rearrangement, ${ }^{47}$ and only one among 29 parents carrying a cryptic translocation ascertained through an unbalanced proband had repeated abortions. $^{34}$

\section{Miscarriages}

To determine whether an abortion is caused by a genetic defect, fibroblasts derived from the skin of spontaneously aborted foetuses are, in some countries routinely, karyotyped. The finding of a chromosomal rearrangement may highlight the presence of a predisposing situation in one of the parents, and the finding of an aneuploidy may confort the parents as recurrence risk is low. Several molecular karyotyping studies on aborted foetuses have detected submicroscopic imbalances. ${ }^{48-50}$ As for postnatal diagnosis, molecular karyotyping may complement or replace the current routine analyses, particularly for those aborted foetuses with normal karyotype. Further research in this area is required.

\section{Mosaicism detection}

When a chromosomal mosaicism is suspected, cultured fibrobasts are karyotyped. It seems likely that molecular karyotyping will enable the detection of such mosaicisms. Further research to confirm that molecular karyotyping enables the detection of such mosaics is required.

\section{Analytical validity}

Analytical validity deals with the technical aspects of molecular karyotyping, that is, the accuracy of a test to detect a chromosomal aberration. Array CGH interrogates thousands of loci in a single experiment. The challenge of an array CGH experiment in a clinical setting is to call all targets without calling either false positives or false negatives. Specificity is defined as the probability of a negative test among patients without disease (those who do not have any chromosomal imbalance), whereas the sensitivity measures the effectiveness of a test to detect a chromosomal imbalance. The ability to detect a chromosomal imbalance depends on the resolution of the array used. To obtain high sensitivity and specificity levels, adequate platforms and adequate threshold algorithms are required, false positive as well as false negative rates have to be determined, and the achievable resolution of the array needs to be established experimentally.

\section{Specificity and sensitivity}

To validate the optimal thresholds and to establish the false positive and the false negative rates of array results in any laboratory setting, a series of experiments including selfself hybridisations, replicate experiments, gender mismatch analyses, hybridisations using DNA with established copy number changes and chromosome add-in experiments should be performed. For example, copy number changes by definition cannot exist in self-self-hybridisations, therefore none of the clones present on the array should report a copy number change. In practice, however, a small number of false calls or flagged reporters (excluded from further data processing) will always be made due to 
labelling bias and hybridisation artefacts. To determine false positive/false negative calling rates and reproducibility, replicate experiments should be performed so that appropriate threshold algorithms can be established minimising false positive/false negative calls. In addition, gender mismatch or hybridisations using DNA with established copy number changes, for example DNA derived from a patient with regular trisomy 21, can support the determination of false positive/false negative rates for that given chromosome-related clones. Therefore the best option would be the use of chromosome-specific add-in samples to validate the hybridisation characteristics of all clones representing the respective chromosomes. ${ }^{51}$ Unfortunately, such add-in samples are not yet commercially available.

While the appearance of a number of consecutive clones with deviating ratios is highly likely to reflect a true copy number change in a patient's genome, single clone calls are difficult to interpret. We therefore suggest including in each array analysis multiple measurements for every target on the array. This could be achieved by either performing replicate (dye-swap) experiments, or providing multiple copies of the same target/redundant targets on the array. Dependent on the probability that an imbalance is causal, follow-up control experiments on the patient and their parents should be performed whenever appropriate. When multiple clones within the same region report a deviation from the expected ratio, the probability that this deviation represents a real imbalance increases. If, on the other hand, only a single target deviates from the expected ratio, this probability decreases. Since probability values will depend on the array quality, the number and the sizes of the targets on an array and in general wet-lab experimental conditions, these probabilities have to be established in each laboratory. This probability can experimentally be established by follow-up validation experiments on a large series of potential imbalances.

In array CGH, DNA from a patient is hybridised against reference control DNA. Different sources of reference DNAs have been used to detect imbalances in patients including DNA from a well-established cell line, DNA from a wellcharacterised reference individual or DNA pooled from a cohort of 'normal' individuals. Another approach, avoiding the need for a specific reference DNA, is the so-called loop hybridisation: DNA from one patient is hybridised against DNA from two other patients. ${ }^{24}$ In this approach it is essential to ensure that all three patients are diagnosed with different phenotypes and do not carry the same imbalance. The choice of the reference DNA is crucial to attribute an imbalance to the reference or to the patient. Possible solutions include replicating the experiment with another reference DNA or use of a very well characterised reference DNA. The development of validated international control samples may be beneficial. However, it should be realised that the importance of reference DNA sample is less relevant by using an SNP array-based approach.

We suggest establishing an internal quality control programme to address these points. Furthermore, if 'inhouse' produced arrays are used for diagnostic purposes, we recommend determining the quality of each printed batch of arrays. This can be performed by hybridising a set number of slides (typically the first and the last) from each batch with patient DNA containing known aberrations. We strongly advise to perform a similar QA programme for commercially available arrays.

\section{Resolution}

The resolution of molecular karyotyping is in the first instance dependent on the platform used. The higher the number of targets, the higher the potential resolution will be. However, the effective resolution will typically be lower because (1) the values of successive targets are often averaged and (2) the average spacing of targets and minimum/maximum coverage of specific regions is variable.

Based on the previously discussed control hybridisations using reference samples with DNA imbalances of variable but well-characterised sizes, the resolution of an array can be determined and should be reported as such. A laboratory can choose to call only those imbalances, which are composed of multiple successive targets and in this way decrease the false positive rate. However, this will also reduce the effective resolution of an array (CGH) experiment. Furthermore, because of the high number of targets on an array, it is almost inevitable that a small number of targets will not respond to copy number changes in the expected way due to, for example, homologous regions or repeats present in the target sequence. This, however, may also reduce the effective resolution of an array and may lead to false negative results. The number of non-responding targets in an otherwise changed region should be documented for each hybridisation. In addition, some areas on the array might not report the expected results due to hybridisation artefacts. These clones should be excluded from the analysis. However, the laboratory should establish a minimal retention threshold for each chromosome as well as for all targets on the array. In our hands a global minimal retention value of $90 \%$ is acceptable. Taken together, a laboratory should determine and report not only the theoretical resolution, but also the experimental resolution of the array to the referring clinician. We strongly recommend that each laboratory participates in external quality control schemes to validate the effective resolution and performance of the arrays used in their laboratories. Such quality control schemes currently only exist within small consortiums. Therefore, there is an urgent need for an organised large-scale effort to develop external quality control schemes. 
Several studies have now shown the ability of molecular karyotyping to detect low-grade mosaicism with aneuploidy as low as $7 \% .^{52,53}$ While in conventional karyotyping the chance to detect such low-grade mosaicism is dependent on the number of metaphases analysed, in array CGH this depends on experimental outcome of the hybridisation, that is the standard deviation of an array experiment as well as on the size and the number of successive called targets. ${ }^{54}$ The degree of mosaicism that can be detected can be established using mixtures of normal DNA versus abnormal DNA.

To validate imbalances detected by array CGH, follow-up analyses might be required. Whether or not alternative techniques are chosen to confirm a specific imbalance depends on the probability that a perceived imbalance is a true positive call (as discussed before). If this probability is low, small imbalances may be verified by independent molecular techniques including FISH, Q-PCR, MLPA or higher resolution arrays. ${ }^{19,20,31,33}$ For deletions, all those techniques will be accurate. For small duplications, FISH should be quantitative, since the resolution of fluorescent microscopes may not separate the signals derived from a duplicon. Since both a deletion and a duplication may result from the unbalanced segregation of a balanced insertional translocation in one of the parents, it is mandatory to perform FISH on the parental metaphase spreads to exclude telomeric/insertional translocations or inversions.

Clone selection and clone validation of mapped sequences used to construct arrays are not considered part of the quality assessment of individual laboratories, but rather belong to the quality management of the array providers. An excellent overview of quality criteria and validation methodology is provided by Fiegler et al..$^{51}$
Both BAC/PAC and oligo-based arrays are successfully used in a clinical diagnostic setting. The maximum resolution of $\mathrm{BAC} / \mathrm{PAC}$ arrays is limited to the insert sizes of the BAC/PACs (in the order of $100-150 \mathrm{~kb}$ ), while the maximum resolution of oligo-based arrays is only limited by the size of the oligos (about $25-70 \mathrm{bp}$ ). However, since in oligo-based arrays consecutive deleted/duplicated targets are required to call an aberration, the true resolution of oligo-based platforms have to be established in each individual laboratory and is often larger than suggested by the array providers. With the highest possible resolution platform, the most genetic information is obtained. However, the higher resolution results at present in interpretation difficulties (see below) and higher resolution platforms are (most often) more expensive. The choice for which resolution, platform and cost/test to implement has to be decided by the individual laboratory. For a technical comparison of the different high-resolution platforms available, we refer to some recent publications. ${ }^{55,56}$

An overview of the main technical problems that can be encountered when performing array CGH as well as their potential causes is provided in Table 1 .

\section{Clinical validity}

In all series of patient studies reported thus far, there are a number of cases in which an aberration could not be linked to the clinical significance. ${ }^{32,57}$ This touches on the difficulties in interpreting aberrations to be causative for a specific phenotype. Currently, causality depends on a number of criteria, which can be considered either alone or in combination. ${ }^{53}$ These include the following.

Table 1 Potential problems in array $\mathrm{CGH}$

\begin{tabular}{|c|c|c|}
\hline Symptoms & Potential problem & Potential solution(s) \\
\hline Low Cy5 signals & $\begin{array}{l}\text { Environmental conditions including } \\
\text { ozone, humidity and temperature }\end{array}$ & $\begin{array}{l}\text { Working in controlled environment, addition of } \\
\text { antioxidants to hybridisation buffers and washing } \\
\text { solutions }\end{array}$ \\
\hline High SD & Low DNA quality & Control of DNA quality \\
\hline High SD & $\begin{array}{l}\text { Insufficient suppression of repeat } \\
\text { sequences }\end{array}$ & Control of Cot1 DNA quality \\
\hline Increased background & Inadequate washing conditions & Adjustment of washing conditions \\
\hline $\begin{array}{l}\text { Fluorescence signal heterogeneity } \\
\text { along the array or spatial biases } \\
\text { introduced by laser misalignment } \\
\text { during image acquisition }\end{array}$ & Poor laser adjustment & $\begin{array}{l}\text { Normalization by sub-array (block-normalization), } \\
\text { adjust laser, proper maintenance of appliance }\end{array}$ \\
\hline $\begin{array}{l}\text { Artifactual ratio changes-labelling } \\
\text { bias }\end{array}$ & Molecular structure of Cy3 and Cy5 & $\begin{array}{l}\text { Indirect labelling approaches using the incorporation } \\
\text { of aminoallyl-modified dNTPs into both DNAs, } \\
\text { followed by independent direct labelling with reactive } \\
\text { fluorochromes. Introduction of replicate dye-reversal } \\
\text { hybridizations or direct chemical labelling by using } \\
\text { ULS- coupled dyes }\end{array}$ \\
\hline Uneven hybridisation & $\begin{array}{l}\text { Manual hybridisation with drying/ } \\
\text { leaking }\end{array}$ & Automated hybridization process \\
\hline Low intensity, high SD & $\begin{array}{l}\text { Low incorporation, poor labelling } \\
\text { efficiency, poor recovery }\end{array}$ & $\begin{array}{l}\text { Strong QC for DNA quality, DOL and labelled DNA } \\
\text { quality for new users }\end{array}$ \\
\hline
\end{tabular}

$\mathrm{CGH}$, comparative genomic hybridisation. 
- The aberration has previously been reported in an individual with the same phenotype. If a recurrent microdeletion/duplication associated with a well-known phenotype is detected, the causal relationship is established. However, most imbalances identified to date are scattered across the genome. Thus far, only a few novel recurrent microdeletion syndromes have been identified. ${ }^{58-61}$ Moreover, in clinical practice, deciding whether the phenotype of a patient is similar to that of a previously reported patient can be difficult, given the fact that the features of the patients are often nonspecific (eg mental handicap, microcephaly, short stature, ...). This however does not apply for specific features such as specific organ malformations. Because most imbalances reported thus far are unique, proof of pathogenicity of the imbalance depends on additional criteria as listed below.

- The presence of a known gene in the aberration which if changed in copy number is known to cause a feature present in the individual. Examples include PMP22, $B W S, A P P, \alpha$-synucleine, CHD7 deletion causing CHARGE syndrome ${ }^{62}$ and DAX1 duplication causing gonadal dysgenesis in $46, \mathrm{XY}$ females. ${ }^{63}$

- A de novo origin of an imbalance is generally thought to be an important argument suggesting the pathogenity of the imbalance. However, given the high number of $\mathrm{CNV}$ in the normal population, one has to consider that some of the de novo detected aberrations may represent a de novo occurrence of a CNV. Nevertheless, current data indicate that few of the known CNVs actually occurred de novo, but most likely have an ancient origin, as seen for SNPs. At present, the frequency by which genomic imbalances occurred de novo is unknown.

- Size of the aberration: Larger imbalances are more likely to be pathogenic. However, cytogeneticists have known for years that even cytogenetically visible imbalances can be benign $^{64}$ and array CGH analyses have sometimes revealed large imbalances $(>5 \mathrm{Mb}$ ), which appeared to be present in phenotypically normal parents of probands. ${ }^{65,66}$ Some of those rare imbalances detected in individuals with MCA/MR, but shown to be inherited from phenotypically normal individuals, can be benign in some individuals but causal in others as the phenotypic expression of certain chromosomal imbalances can vary. One possible (but rare) mechanism is the unmasking of a recessive allele. ${ }^{67}$ Another is deletion/ duplication of imprinted regions of the genome. Probably more frequent is the existence of genetic and environmental modifiers. Therefore, inherited imbalances may well turn out to be susceptibility loci contributing to pathogenicity. ${ }^{57}$ An example is the 1q21.1 microdeletion associated with TAR syndrome. ${ }^{68}$ More frequent situations are X-linked imbalances inherited from a normal mother or autosomal imbalances of an imprinted region inherited from a normal parent in which the unbalanced region is imprinted.
Because of these interpretation difficulties, the success of introducing array $\mathrm{CGH}$ in the clinical practice will depend to a large extent on the availability of accurate genotype/ phenotype correlations on a large number of chromosomal aberrations. Efforts to establish genome-wide data are currently ongoing (DECIPHER ${ }^{69}$ and ECARUCA ${ }^{70}$ ) and are already available to the genetics community. At present, counselling patients with perceived de novo/pathogenic or benign/inherited imbalances should be performed with caution and in collaboration between cytogeneticists and clinical geneticists.

\section{Clinical implementation}

Molecular karyotyping should be performed in wellorganised and specialised laboratory facilities that provide cytogenetic, and/or molecular genetic testing and are supervised by laboratory specialists trained in clinical cytogenetics and/or clinical molecular genetics. The director, laboratory supervisors and technical staff must have adequate qualifications, education and experience regarding their position. The laboratory facilities should also have access to medical expertise on a regular basis and maintain research and/or scientific collaborations. Laboratory facilities must provide the appropriate working environment and equipment suitable for the technology. Prior to the provision of diagnostic molecular karyotyping, the laboratory should validate the methodology with known large and small sized imbalances. The laboratory should have written laboratory protocols, and procedures for molecular karyotyping and related procedures in the standard operating procedures manual. For both, clinicians and laboratory staff, training should be organised with regard to technical aspects, bioinformatics and data interpretation.

\section{Consent for analysis and ethical considerations}

Due to the high frequency of CNV in the human genome, high-resolution array CGH of DNA from a normal individual reveals an individual 'signature' of copy number variants. ${ }^{50}$ Thus, if high-resolution array CGH of parental samples is used to determine whether copy number variants are de novo or inherited, the potential to reveal misattributed parentage, for example non-paternity, is high. Homozygosity mapping using SNP arrays has been a powerful technology for identifying novel autosomal recessive genes in highly consanguineous families. When applied to 'molecular karyotyping' the potential for SNP arrays to reveal consanguinity should be kept in mind. These aspects should be considered by the clinician when obtaining consent for high-resolution molecular karyotype analysis. One has to realize that this guideline is only a scientific recommendation; testing and reporting should be in line with the national ethical/legal regulations, especially when it concerns prenatal testing, counselling and storage of genotyping information. 


\section{Array reporting}

The report should include, as is currently the case for conventional karyotyping, information for laboratory identification, patient identification using two different identifiers, referring physician/scientist identification, sample information (type of sample, date of referral, date of report and unique sample identification) and referral information (reason for referral and clinical indication of test) where appropriate. ${ }^{71}$ In addition, information on the methodology used for molecular karyotyping and the array resolution level should be presented. The molecular karyotyping result should be presented using the ISCN 2005 nomenclature. ${ }^{72}$ In addition, a written description and interpretation of the result is important. It is the responsibility of the laboratory to provide a clearly understandable report for the referring clinician/scientist. The report must include the results and the interpretation with the possible clinical implications. Interpretation of results requires supervision by an appropriately trained physician or scientist. In cases where the quality or level of the analysis fails to achieve agreed standards, the report should explain the limitations of the results. The most important features that must be reported are summarised in Table 2.

Laboratory reporting times should take into account the reason for referral and level of urgency. Laboratory report times should be kept as short as possible and time should be reasonable.

The introduction of expression arrays interrogating tens of thousands of genes confronted researchers with the need to report data in a uniform and easily accessible format. To this end, the members of the MGED Society (Microarray Gene Expression Data) have worked with the broader scientific community and developed standards for annota- tion and exchange of microarray data (http://www.mged. org). A document called Minimum Information about a Microarray Experiment, ${ }^{73}$ also known as the MIAME guidelines' was developed as the basis for microarray data standards. Similarly, these MIAMI guidelines can define the basic information derived from array CGH experiments that needs to be stored. In short, the experimental conditions of the array experiments as well as image analysis files (ie GPR files) and data interpretation files need to be stored digitally and/or in the patient records.

\section{Concluding remarks}

Array CGH is a technology that is rapidly evolving. Novel and increasingly higher resolution genome-wide screening tools are currently being developed. In addition to both clinical utility and validity, there is a cost/benefit relationship that influences the clinical introduction of novel technologies. These issues have been addressed elsewhere. $^{74}$ Our understanding of benign and pathogenic genomic variation is still in its infancy, and therefore both clinicians and cytogeneticists are encouraged to contribute information to Databases such as DECIPHER and ECARUCA to increase the knowledge on phenotype/genotype correlations. These efforts will change our knowledge dramatically in the years to come. This article therefore, by necessity, aims to provide best operational guidelines at the current state with regard to current technology and our biological understanding. The working group conceives that in the coming years, this document will need to be updated in a regular manner.

Table 2 In-house laboratory report

\author{
Platform used \\ Number of reporters on the array \\ Achievable resolution \\ Reference DNA \\ Size of the imbalance \\ Flanking normal reporters \\ First and last significantly aberrant reporters \\ Total number of aberrant reporters \\ ISCN 2005 \\ De novo or inherited (paternal or maternal inherited) \\ Independent validation by other method \\ Optional include schematic representation (print) of aberrant region \\ for illustration \\ CNVs are documented but do not need to be reported
}

Refer to technical protocol/SOP and version used

If possible, provide biological information about genes in the imbalance, known phenotypes, etc.

Caution
Genome wide or targeted

Average, minimum and maximum regions Optional

Indicate version of genome Browser used

Optional

If investigated

Specify (ie name, position and number of probes used for validation)

'Further data regarding presumed normal copy number variation was obtained by this analysis and is available upon request ${ }^{\prime}$

'The SOP is accessible to referring clinicians upon request'

'This is an emerging field, just best effort!!'

CNVs, copy number variations; SOP, standard operating procedure. 


\section{Acknowledgements}

We thank EuroGentest Network of Excellence Project 2005-EU Contract no. FP6-512148 for support of the initial meeting leading to this article. We also thank Jean-Jacques Cassiman and Jean-Pierre Fryns for their critical reading of the manuscript.

\section{References}

1 Ford CE, Hamerton JL: The chromosomes of man. Nature 1956; 178: $1020-1023$.

2 Tjjio JH, Levan A: The chromosome number of man. Hereditas 1956; 42: $1-6$.

3 Ford CE, Jones KW, Polani PE, De Almeida JC, Briggs JH: A sexchromosome anomaly in a case of gonadal dysgenesis (Turner's syndrome). Lancet 1959; 1: 711-713.

4 Jacobs PA, Strong JA: A case of human intersexuality having a possible XXY sex-determining mechanism. Nature 1959; 183: $302-303$.

5 Lejeune J, Gautier M, Turpin R: Etudese des chromosomes somatiques de neuf enfants mongoliens. C R Acad Sci 1959; 248: $1721-1722$.

6 Caspersson T, Zech L, Modest EJ: Fluorescent labeling of chromosomal DNA: superiority of quinacrine mustard to quinacrine. Science 1970; 170: 762

7 Drets ME, Shaw MW: Specific banding patterns of human chromosomes. Proc Natl Acad Sci USA 1971; 68: 2073-2077.

8 Schinzel A: Catalogue of Unbalanced Chromosome Aberrations in Man. Berlin/New York: Walter de Gruyter, 2001.

9 Yunis JJ: High resolution of human chromosomes. Science 1976; 191: $1268-1270$.

10 Speicher MR, Carter NP: The new cytogenetics: blurring the boundaries with molecular biology. Nat Rev Genet 2005; 6: $782-792$.

11 Sellner LN, Taylor GR: MLPA and MAPH: new techniques for detection of gene deletions. Hum Mutat 2004; 23: 413-419.

12 Speicher MR, Gwyn BS, Ward DC: Karyotyping human chromosomes by combinatorial multi-fluor FISH. Nat Genet 1996; 12: $368-375$.

13 Szuhai K, Tanke HJ: COBRA: combined binary ratio labeling of nucleic-acid probes for multi-color fluorescence in situ hybridization karyotyping. Nat Protocols 2006, Published online 27 June 2006; doi:10.1038/nprot.2006.41. http://www.nature.com/nprot/ journal/v1/n1/pdf/nprot.2006.41.pdf.

14 Kallioniemi A, Kallioniemi OP, Sudar D et al: Comparative genomic hybridization for molecular cytogenetic analysis of solid tumors. Science 1992; 258: 818-821.

15 Pinkel D, Segraves R, Sudar D et al: High resolution analysis of DNA copy number variation using comparative genomic hybridization to microarrays. Nat Genet 1998; 20: 207-211.

16 Solinas-Toldo S, Lampel S, Stilgenbauer S et al: Matrix-based comparative genomic hybridization: biochips to screen for genomic imbalances. Genes Chromosomes Cancer 1997; 20: 399-407.

17 Albertson DG, Pinkel D: Genomic microarrays in human genetic disease and cancer. Hum Mol Genet 2003; 12: R145-R152.

18 Cai WW, Mao JH, Chow CW, Damani S, Balmain A, Bradley A: Genome-wide detection of chromosomal imbalances in tumors using BAC microarrays. Nat Biotechnol 2002; 20: 393-396.

19 Shaw-Smith C, Redon R, Rickman L et al: Microarray based comparative genomic hybridisation (array-CGH) detects submicroscopic chromosomal deletions and duplications in patients with learning disability/mental retardation and dysmorphic features. J Med Genet 2004; 41: 241-248.

20 Vissers LE, de Vries BB, Osoegawa K et al: Array-based comparative genomic hybridization for the genomewide detection of submicroscopic chromosomal abnormalities. Am J Hum Genet 2003; 73: $1261-1270$
21 Vermeesch JR, Rauch A: Reply to Hochstenbach et al. Eur J Hum Genet 2006; 14: 1063-1064.

22 Veltman JA: Genomic microarrays in clinical diagnosis. Curr Opin Pediatr 2006; 18: 598-603.

23 Friedman JM, Baross A, Delaney AD et al: Oligonucleotide microarray analysis of genomic imbalance in children with mental retardation. Am J Hum Genet 2006; 79: 500-513.

24 Menten B, Maas N, Thienpont B et al: Emerging patterns of cryptic chromosomal imbalance in patients with idiopathic mental retardation and multiple congenital anomalies: a new series of 140 patients and review of published reports. J Med Genet 2006; 43: 625-633.

25 Burke W: Genetic testing. N Engl J Med 2002; 347: 1867-1875.

26 Roeleveld N, Zielhuis GA, Gabreels F: The prevalence of mental retardation: a critical review of recent literature. Dev Med Child Neurol 1997; 39: 125-132.

27 Rauch A, Hoyer J, Guth S et al: Diagnostic yield of various genetic approaches in patients with unexplained developmental delay or mental retardation. Am J Med Genet A 2006; 140: 2063-2074.

28 Christianson A, Howson CP, Modell B: March of Dimes Global Report on Birth Defects - The hidden Toll of Dying and Disabled Children. White Plains, New York: March of Dimes Birth Defects Foundation, 2006

29 Johnson CP, Walker Jr WO, Palomo-Gonzalez SA, Curry CJ: Mental retardation: diagnosis, management, and family support. Curr Probl Pediatr Adolesc Health Care 2006; 36: 126-165.

30 Bauters M, Van Esch H, Marynen P, Froyen G: X chromosome array-CGH for the identification of novel X-linked mental retardation genes. Eur J Med Genet 2005; 48: 263-275.

31 Schoumans J, Ruivenkamp C, Holmberg E, Kyllerman M, Anderlid BM, Nordenskjold M: Detection of chromosomal imbalances in children with idiopathic mental retardation by array based comparative genomic hybridisation (array-CGH). J Med Genet 2005; 42: 699-705.

32 Rosenberg C, Knijnenburg J, Bakker E et al: Array-CGH detection of micro rearrangements in mentally retarded individuals: clinical significance of imbalances present both in affected children and normal parents. J Med Genet 2006; 43: 180-186.

33 de Vries BB, Pfundt R, Leisink $\mathrm{M}$ et al: Diagnostic genome profiling in mental retardation. Am J Hum Genet 2005; 77: 606-616.

34 Ravnan JB, Tepperberg JH, Papenhausen P et al: Subtelomere FISH analysis of 11688 cases: an evaluation of the frequency and pattern of subtelomere rearrangements in individuals with developmental disabilities. J Med Genet 2006; 43: 478-489.

35 Ciccone R, Giorda R, Gregato G et al: Reciprocal translocations: a trap for cytogenetists? Hum Genet 2005; 117: 571-582.

36 Gribble SM, Prigmore E, Burford DC et al: The complex nature of constitutional de novo apparently balanced translocations in patients presenting with abnormal phenotypes. J Med Genet 2005; 42: $8-16$.

37 Knijnenburg J, van Haeringen A, Hansson KB et al: Ring chromosome formation as a novel escape mechanism in patients with inverted duplication and terminal deletion. Eur I Hum Genet 2007; 15: 548-555.

38 Larrabee $\mathrm{PB}$, Johnson KL, Lai $\mathrm{C}$ et al: Global gene expression analysis of the living human fetus using cell-free messenger RNA in amniotic fluid. JAMA 2005; 293: 836-842.

39 Miura S, Miura K, Masuzaki H et al: Microarray comparative genomic hybridization (CGH)-based prenatal diagnosis for chromosome abnormalities using cell-free fetal DNA in amniotic fluid. J Hum Genet 2006; 51: 412-417.

40 Rickman L, Fiegler H, Shaw-Smith C et al: Prenatal detection of unbalanced chromosomal rearrangements by array CGH. J Med Genet 2006; 43: 353-361.

41 Sahoo T, Cheung SW, Ward P et al: Prenatal diagnosis of chromosomal abnormalities using array-based comparative genomic hybridization. Genet Med 2006; 8: 719-727.

42 Warburton D: De novo balanced chromosome rearrangements and extra marker chromosomes identified at prenatal diagnosis: 
58 Koolen DA, Vissers LE, Pfundt R et al: A new chromosome 17q21.31 microdeletion syndrome associated with a common inversion polymorphism. Nat Genet 2006; 38: 999-1001.

59 Shaw-Smith C, Pittman AM, Willatt L et al: Microdeletion encompassing MAPT at chromosome $17 \mathrm{q} 21.3$ is associated with developmental delay and learning disability. Nat Genet 2006; 38: $1032-1037$.

60 Sharp AJ, Hansen S, Selzer RR et al: Discovery of previously unidentified genomic disorders from the duplication architecture of the human genome. Nat Genet 2006; 38: 1038-1042.

61 Menten B, Buysse K, Zahir F et al: Osteopoikilosis, short stature and mental retardation as key features of a new microdeletion syndrome on 12q14. J Med Genet 2007; 44: 264-268.

62 Vissers LE, Van Ravenswaaij CM, Admiraal R et al: Mutations in a new member of the chromodomain gene family cause CHARGE syndrome. Nat Genet 2004; 36: 955-957.

63 Barbaro M, Oscarson M, Schoumans J, Staaf J, Ivarsson SA, Wedell A: Isolated 46,XY gonadal dysgenesis in two sisters caused by a Xp21.2 interstitial duplication containing the DAX1 gene. J Clin Endocrinol Metab 2007, e-pub ahead of print.

64 Barber JC: Directly transmitted unbalanced chromosome abnormalities and euchromatic variants. I Med Genet 2005; 42: $609-629$.

65 Balikova I, Menten B, de Ravel T et al: Subtelomeric imbalances in phenotypically normal individuals. Hum Mutat 2007, e-pub ahead of print.

66 Hansson K, Szuhai K, Knijnenburg J, van Haeringen A, de Pater J: Interstitial deletion of $6 \mathrm{q}$ without phenotypic effect. Am J Med Genet A 2007; 143: 1354-1357.

67 Lesnik Oberstein SA, Kriek M, White SJ et al: Peters Plus syndrome is caused by mutations in B3GALTL, a putative glycosyltransferase. Am I Hum Genet 2006; 79: 562-566.

68 Klopocki E, Schulze H, Strauss G et al: Complex inheritance pattern resembling autosomal recessive inheritance involving a microdeletion in thrombocytopenia-absent radius syndrome. Am J Hum Genet 2007; 80: 232-240.

69 DECIPHER: DatabasE of Chromosome Imbalance and Phenotype in Humans Using Ensembl Resources, http://decipher.sanger.uk, 2007.

70 Feenstra I, Fang J, Koolen DA et al: European cytogeneticists association register of unbalanced chromosome aberrations (ECARUCA); an online database for rare chromosome abnormalities. Eur J Med Genet 2006; 49: 279-291.

71 Bricarelli FD, Cavani S, Hastings RJ, Kristoffersson U: Cytogenetics guidelines and quality assurance. ECA Newsletter 2006; 17: $13-32$.

72 Shaffer L, Tommerup N: ISCN 2005. Basel: Karger, 2005.

73 Brazma A, Hingamp P, Quackenbush J et al: Minimum information about a microarray experiment (MIAME)-toward standards for microarray data. Nat Genet 2001; 29: 365-371.

74 Newman WG, Hamilton S, Ayres J et al: Array comparative genomic hybridization for diagnosis of developmental delay - an exploratory cost-consequences analysis. Clin Genet 2007; 71 (3): $254-271$. 